\title{
Whose hand is this? Person Identification from Egocentric Hand Gestures
}

\author{
Satoshi Tsutsui \\ Indiana University \\ Bloomington, IN, USA \\ stsutsuidindiana.edu
}

\author{
Yanwei Fu \\ Fudan University \\ Shanghai, China \\ yanweifu@fudan.edu.cn
}

\author{
David Crandall \\ Indiana University \\ Bloomington, IN, USA \\ djcraneindiana.edu
}

\begin{abstract}
Recognizing people by faces and other biometrics has been extensively studied in computer vision. But these techniques do not work for identifying the wearer of an egocentric (first-person) camera because that person rarely (if ever) appears in their own first-person view. But while one's own face is not frequently visible, their hands are: in fact, hands are among the most common objects in one's own field of view. It is thus natural to ask whether the appearance and motion patterns of people's hands are distinctive enough to recognize them. In this paper, we systematically study the possibility of Egocentric Hand Identification (EHI) with unconstrained egocentric hand gestures. We explore several different visual cues, including color, shape, skin texture, and depth maps to identify users' hands. Extensive ablation experiments are conducted to analyze the properties of hands that are most distinctive. Finally, we show that EHI can improve generalization of other tasks, such as gesture recognition, by training adversarially to encourage these models to ignore differences between users.
\end{abstract}

\section{Introduction}

Vision-based person identification has many practical applications in safety and security applications, so developing techniques to identify individual people is among the most-studied computer vision problems [22]. After decades of research, some vision-based biometric technologies are now commonplace, with retina and and palm recognition routinely used in high-security applications such as border control [11], while even consumer smartphones feature fingerprint [25] and face recognition [12]. Other techniques that identify more subtle distinguishing features, such as gait [47] or keystroke dynamics [5], have also been used in some applications.

User identification for egocentric cameras presents an interesting challenge, however, because the face and body of the camera wearer are rarely seen within their own first-

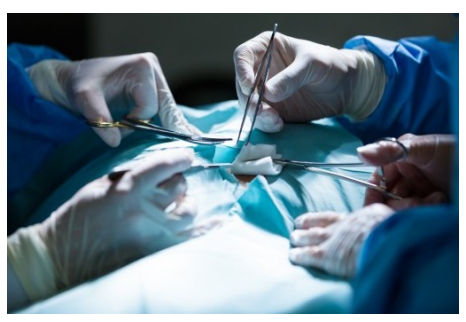

(a) Medical surgery with hands [1]

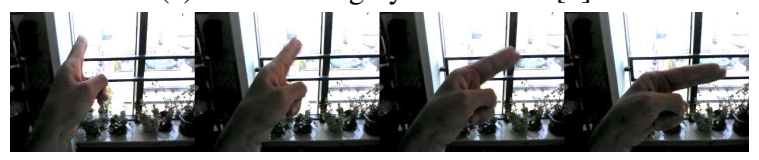

(b) RGB frames



(c) Depth frames

Figure 1: (a) If multiple people are collaborating on a task assisted by VR devices, can we tell whose hand is whose in the first person view? $(b, c)$ Sample input frames. Our results suggest that even egocentric depth data can give evidence about who is whom.

person field of view. There is a notable exception, however: we use our hands as a primary means of manipulating, interacting with, and sensing the physical world around us, and thus our hands are frequently in our field of view [4]. In fact, our own hands may be the objects that we see most frequently throughout the entire span of our lives and thus may even play a special role in our visual systems, including in how children develop object perception [41].

We hypothesize that it is possible to recognize individuals based only on their hand appearances and motion patterns. If correct, this hypothesis would have numerous potential applications. A VR/AR device shared among members of a household could tailor its behavior to a specific user's preferences. Some wearable cameras need user au- 
thentication (e.g., verifying the wearer of a police body camera [42]), and this could provide one signal. When multiple people are interacting or collaborating on a manual task, such as surgery (Figure 1a), the field of view may be full of hands, and understanding the activities in the scene would involve identifying whose hand is whose.

Some existing work has studied using properties of hands, such as shape, to uniquely identify people, including classic papers that use manually-engineered features [38, 48]. However, these techniques require hand images with a clearly visible palm, and are not applicable for unconstrained egocentric videos.

This paper, to our knowledge for the first time, studies the task of Egocentric Hand Identification (EHI). Since this is the first work, we focus on establishing baselines and analyzing which factors of the egocentric video contribute to the recognition accuracy. Specifically, we demonstrate that standard computer vision techniques can learn to recognize people based on egocentric hand gestures with reasonable accuracy even from depth images alone. We then conduct ablation studies to determine which feature(s) of inputs are most useful for identification, such as 2D/3D shape, skin texture (e.g. moles), and skin color. We do this by measuring recognition accuracy as a function of manipulations of the input video; e.g. we prepare gray-scale video of hand gestures (Figure 2(4)), and binary-pixel video composed of hand silhouettes only (Figure 2 (3)), and regard the accuracy increase between gray-scale videos and hand silhouettes as the contribution of skin texture. Our results indicate that all these elements (2D hand shape, 3D hand shape, skin texture, and skin color) carry some degree of distinctive information.

We further conjectured that this task of hand identification could improve other related tasks such as gesture recognition. However, we found that the straightforward way of doing this - training a multi-task CNN to jointly recognize identity and gesture - was actually harmful for gesture recognition. While perhaps surprising at first, this result is intuitive: we want the gesture classifier to generalize to unseen subjects, so gesture representations should be predictive of gestures but invariant to the person performing gestures. We use this insight to propose an adversarial learning framework that directly enforces this constraint: we train a primary classifier to perform gesture recognition while also training a secondary classifier to fail to recognize who performs gestures. Our experiments indicate that the learned representation is invariant to person identity, and thus generalizes well for unseen subjects.

In summary, the contributions of this paper are as follows:

1. To our knowledge, we are the first to investigate if it is possible to identify people based only on egocentric videos of their hands.
2. We perform ablation experiments and investigate which properties of gesture videos are key to identifying individuals.

3. We propose an adversarial learning framework that can improve hand gesture recognition by explicitly encouraging invariance across person identities.

\section{Related Work}

Egocentric vision (or first-person vision) develops computer vision techniques for wearable camera devices. In contrast to the third-person perspective, first-person cameras have unique challenges and opportunities for research. Previous work on egocentric computer vision has concerned object recognition [6, 26, 28], activity recognition [3, 10, 29, 31, 34, 37, 39, 50], gaze prediction [21, 27, 43, 51], video summarization [19, 35, 40], head motion signatures [36], and human pose estimation [23,49]. In particular, many papers have considered problems related to hands in egocentric video, including hand pose estimation [16,30], hand segmentation [4 46], handheld controller tracking [33], and hand gesture recognition [8]. However, to our knowledge, no prior work has considered egocentric hand-based person identification. The closest work we know of is extracting user identity information from optical flow [20, 44], which is complementary to our work - integrating optical flow into our study would be future work.

In a broad sense, using hands to identify people has been well-studied, particularly for fingerprint identification [25], but it is not realistic to assume that we can extract fingerprints from egocentric video. Outside of biometrics, some classic papers have shown the potential to identify subjects based on hand shapes and geometries. Sanchez-Reillo et al. use palm and lateral views of the hand, and define features based on widths and heights of predefined key points [38]. Boreki et al. propose 25 geometric features of the finger and palm [7], while Yoruk et al. use independent component analysis on binary hand silhouettes [48]. However, these studies use very clean scans of hands with the palm clearly visible, often with special scanning equipment specifically designed for the task [38]. Views of hands from wearable cameras are unconstrained, much noisier, and capture much greater diversity of hand poses, so these classic methods are not applicable. Instead, we build on modern computer vision to learn features effective for this task in a data-driven manner.

We are aware of two studies that apply modern computer vision for hand identification. Mahmoud [2] applies CNNs for learning representations from controlled hand images with the palm clearly visible. Uemori et al. use multispectral images of skin patch from hands, and train 3D CNNs to classify the subjects [45]. The underlying assumption for this approach is that each individual has distinctive 


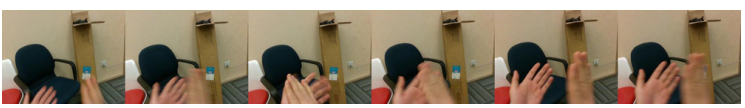

(1) Original RGB

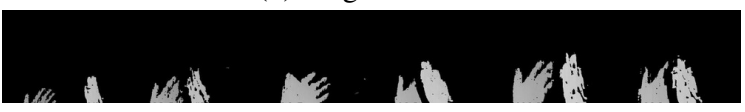

(2) Original Depth.

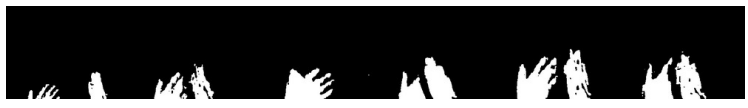

(3) Binary Hand: Binarized depth map that approximates the shapes of hands.



(4) Gray Hand: Grayscale images masked with hands.



(5) Color Hand: Color images with hands.

Figure 2: Controlled Inputs

skin spectra due to the unique chromophore concentrations within their skin [14]. Although this method does not have any constraints for hand pose, it requires a multispectral sensors, which are not usually available in consumer devices. Moreover, it assumes images of the hands are clear, not blurry, and with good lighting conditions, which are not practical assumptions for egocentric vision.

\section{Methodology}

Our goal is to investigate if it is possible to identify a camera wearer from an egocentric video clip of them performing hand gestures. Our goal is not to engineer a new method but to investigate whether a simple end-to-end technique can learn to perform this task. Specifically, we build upon a standard convolutional neural network [17] for video classification, and train an end-to-end subject classifier from video clips. Our approach uses the raw inputs from RGB or depth sensors directly, and intentionally does not employ hand-pose or hand-mesh recognition because we do not want our method to depend on the recognition results of other tasks (which would complicate the method and make it vulnerable to failures of those subtasks).

\subsection{Egocentric Hand Identification (EHI)}

Our focus is to understand the influence of various sources of evidence - RGB, depth, etc. - on the classification decisions. This is important due to the data-driven nature of deep learning and difficulty in collecting largescale egocentric video datasets that are truly representative of all people and environments: it is possible for a classifier to cheat by learning bias of the training data (e.g., if all gestures of a subject are recorded in the same room, the classifier could memorize the background). We try to avoid this by making sure the training and testing videos are recorded in different places, but we also try to identify the types of features (e.g. hand shapes, skin texture, skin color) that are important for CNNs to identify the subjects. In order to factor out each element, we ablate the input video and gradually increase information starting from the silhouettes of hands to the full-color images of hands. In the remainder of this section, we discuss the above-mentioned points in detail, starting from the RGB and Depth inputs available to us.

RGB. We have RGB video clips of gestures against various backgrounds. The RGB data implicitly captures $2 \mathrm{D}$ hand shape, skin texture, and skin color. We show a sample clip in Figure 2 (1), containing not only hands but also a doll. In fact, the same doll appears in other gesture videos of the same subject, which is problematic if the person classification model learns to rely on this background information. This is not just a dataset problem, but a practical problem for possible real-world applications of egocentric vision: a user may record videos in their room to register their hands in the system but still want the device to recognize hands elsewhere. To simulate this point, we train on indoor videos and evaluate on outdoor videos.

Depth. We also have depth video synchronized with the RGB clips (Figure 2(2)). The depth images contain information about 3D hand shape in addition to shapes of objects in the background. A clear advantage of using depth is that it allows for accurate depth-based segmentation of the hands from the background, just by thresholding on distance from the camera. Although less sensitive to the background problem, there is still a chance that depth sensors capture the geometries of the background (e.g. rooms). In order to eliminate background, we apply Otsu's binarization algorithm [32] to separate the background and foreground. It is reasonable to assume that hands are distinctively closer to the device, so the foreground mask corresponds to binary hand silhouettes - an approximation of the hand shapes without 3D information. These hand silhouettes are a starting point for our ablative study.

Binary Hand. We obtain binary hand silhouettes by binarizing the depth maps as discussed above. We show an example in 2(3). This only contains hand shape information and is the input with the least information in our study. We prepare several other inputs by adding more information to this binary video and use the accuracy gain to measure the contribution of additional information, as described below.

3D Hand. We apply the binary mask to the depth images and extract the depth corresponding to the hand region only. The accuracy increase from Binary Hand is a measure of the 
importance of 3D hand shape for identifying the subjects.

Gray Hand. We apply the binary mask to the grayscale frame converted from RGB; see example in Figure 2 (4). This adds hand texture information to the 2D Hands. The accuracy gain from Binary Hand indicates the importance of textures of hands (including moles and nails).

Color Hand. We extract the hand from RGB frames, eliminating the background. We show an example in Figure 2 (5). The accuracy gain from Gray Hand is the contribution of skin color.

3D Color Hand. This is the combination of all available information where hands are extracted from RGBD frames. The accuracy indicates synergies of $2 \mathrm{D}$ hand shape, $3 \mathrm{D}$ hand shape, skin texture, and skin color.

\subsection{Improving gesture recognition}

We hypothesize that our technique is not only useful for identifying people from hands, but also for assisting in other gesture recognition problems. This hypothesis is based on results on other problems that show that multi-task training can perform each individual task better, perhaps because the multiple tasks help to learn a better feature representation. A naive way to implement the idea of jointly estimating gesture and identity is to train gesture classification together with subject classifier, sharing the feature extraction $\mathrm{CNN}$. Then, we can minimize a joint loss function,

$$
\min _{\left(F, C_{g}, C_{p}\right)}\left(\mathcal{L}_{g}+\mathcal{L}_{p}\right)
$$

where $F, C_{g}, C_{p}, \mathcal{L}_{g}, \mathcal{L}_{p}$ are the shared CNNs for feature extraction, the classifier to recognize the gestures, the classifier to recognize identity, the loss for gesture recognition, and the loss for recognizing subjects, respectively.

However, and somewhat surprisingly, we empirically found that minimizing this joint loss decreases the accuracy of gesture recognition. Our interpretation is that because the gesture classification is trained and tested on disjoint subjects, learning a representation that is predictive of person identity is harmful in classifying the gestures performed by new subjects. We hypothesized that a model trained for the "opposite" task, learning a representation invariant to identity, should better generalize to unseen subjects. This can be expressed as a min-max problem of the two tasks,

$$
\min _{\left(F, C_{g}\right)} \max _{C_{p}}\left(\mathcal{L}_{g}+\mathcal{L}_{p}\right)
$$

Intuitively, this equation encourages the $\mathrm{CNN}$ to learn the joint representation $(F)$ that can predict gestures $\left(C_{g}\right)$ but cannot predict who performs the gestures $\left(C_{p}\right)$. This min-max problem is the same as that used in adversarial domain adaptation [15], because the gesture classifier is trained adversarially with the subject classifier. Following the original method [15], we re-write equation 2 into an alternating optimization of two equations,

$$
\begin{gathered}
\min _{\left(F, C_{g}\right)}\left(\mathcal{L}_{g}-\lambda \mathcal{L}_{p}\right) \\
\max _{C_{p}} \mathcal{L}_{p}
\end{gathered}
$$

where $\lambda$ is a hyper-parameter.

\section{Experiments}

We perform our primary experiments on the EgoGesture [8, 52] dataset, which is a large dataset of hands taken both indoors and outdoors. We also perform secondary experiments on the relatively small Green Gesture (GGesture) [9] dataset.

Implementation Details. We use ResNet18 [18] with 3D convolutions [17]. We initialize the model with weights pretrained from the Kinetics dataset, and fine-tune with minibatch stochastic gradient descent using Adam [24] with batch size of 32 and initial learning rate of 0.0001 . We iterate over the dataset for 20 epochs and decrease the learning rate by a factor of 10 at the 10 th epoch. The spatiotemporal input size of the model is $112 \times 112 \times 16$, corresponding to width, height, and frame lengths respectively. In order to fit arbitrary input clips into this resolution, we resize the spatial resolution into $171 \times 128$ via bilinear interpolation, then apply random crops for training and center crops for testing. For the temporal dimension, we train with randomlysampled clips of 16 consecutive frames and test by averaging the prediction of 16 consecutive sliding windows with 8 overlapping frames. If the number of frames is less than 16, we pad to 16 by repeating the first and last frames.

\subsection{Experiments on EgoGesture}

The EgoGesture [8, 52] dataset includes 24,161 egocentric video clips of 83 gesture classes performed by $50 \mathrm{sub}$ jects, recorded both indoors and outdoors. Our task is to classify each video into one of 50 subjects. As discussed in $\mathrm{Sec} 3.1$, we use indoor videos for training, and outdoor videos for evaluation, so that we can prevent leaking user identities based on the backgrounds. The dataset has 16,373 indoor clips, which are used for training, and 7,788 outdoor clips of which we use 3,892 for validation and 3,896 for testing.

\subsubsection{Subject Recognition Results}

We summarize the subject recognition results in Table 1 The original inputs of RGB and Depth can achieve accuracies $(\%)$ of 6.29 and 11.47 . Because a random guess baseline is $2 \%$, our results indicate the potential to recognize people based on egocentric hand gestures. In order to understand where this information comes from, we factor the 


\begin{tabular}{|c|c|c|c|c|c|c|c|}
\hline & \multicolumn{5}{|c|}{ Information } & \multicolumn{2}{|c|}{ Accuracy } \\
\hline & 2D Shape & 3D Shape & Skin Texture & Skin Color & Background & EgoGesture & GGesture \\
\hline RGB & $\checkmark$ & - & $\checkmark$ & $\checkmark$ & $\checkmark$ & 6.29 & 61.09 \\
\hline Depth & $\checkmark$ & $\checkmark$ & - & - & $\checkmark$ & 11.47 & - \\
\hline Binary Hand & $\checkmark$ & - & - & - & - & 11.11 & 51.13 \\
\hline 3D Hand & $\checkmark$ & $\checkmark$ & - & - & - & 12.04 & - \\
\hline Gray Hand & $\checkmark$ & - & $\checkmark$ & - & - & 14.22 & 61.09 \\
\hline Color Hand & $\checkmark$ & - & $\checkmark$ & $\checkmark$ & - & 18.53 & 64.71 \\
\hline 3D Color Hand & $\checkmark$ & $\checkmark$ & $\checkmark$ & $\checkmark$ & - & 19.53 & - \\
\hline
\end{tabular}

Table 1: Subject recognition accuracy (\%) for each ablated input on EgoGesture dataset and GGesture dataset.



(1) A training gesture clip recorded indoor.



(2) CAM showing that prediction is cuing on background.

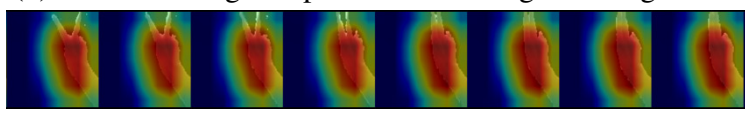

(3) CAM focuses on hands if we mask out the background.

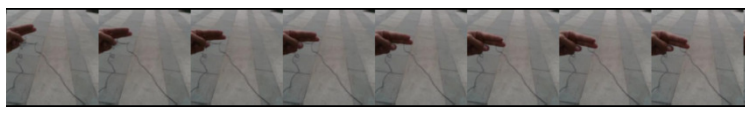

(4) A test clip recorded outdoors.

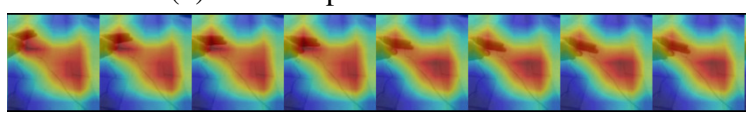

(5) CAM focusing on background.

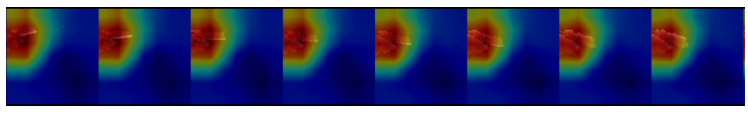

(6) CAM focuses on hands when background is masked out.

Figure 3: Class Activation Maps (CAMs)

input into different components and discuss the results below.

The accuracy on Binary Hand is $11.11 \%$. This input contains only hand silhouettes, indicating that it is possible to recognize a person's identity to some degree using $2 \mathrm{D}$ shape alone. This is the starting point of our analysis as we gradually add additional information. Adding depth information to the binary mask (3D hand) increases the accuracy to $12.04 \%$. This indicates that $3 \mathrm{D}$ information, including the distance from a camera to hands, contributes to better identify the subjects. The gray-scale images of hands (Gray

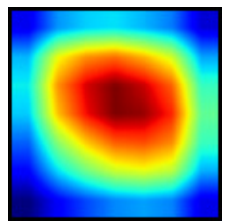

(a) RGB

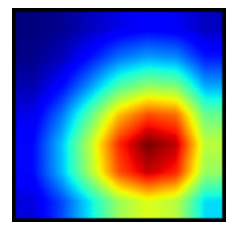

(b) Color Hand

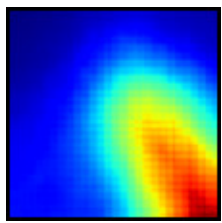

(c) Actual Hand
Figure 4: (a) Average CAM produced by the model trained from raw images with hand and background. (b) Average CAM produced by the model trained from the images only with hand. (c) Average of the actual hand masks. Overall, CAM by the hand-only model has its peak closer to the actual hand location.

Hands) achieve an accuracy of $14.22 \%$, suggesting that the texture of skin carries some information. RGB images of hands (Color Hand) can achieve an accuracy of $18.53 \%$, suggesting that skin color also conveys information about personal identity.

The results so far show that 2D shapes of hands, 3D shapes of hands, skin texture, and skin color carry information about the identity of a subject. We can combine all of them with RGBD frames masked with hand segmentation maps (3D Color Hand), and this achieves the highest accuracy of $19.53 \%$. This indicates that each property contributes at least some additional, complementary information.

\subsubsection{Background overfitting via CAM Visualization}

In terms of unmodified inputs, the accuracy of RGB $(6.29 \%)$ is significantly worse than depth $(11.47 \%)$. This is somewhat surprising given that our ablative study shows that RGB images only with hands have much higher accuracy $(18.53 \%)$ and that skin color and texture information are important cues. We suspect the reason lies in the way we design the task: because we train on indoor videos and 


\begin{tabular}{lcc}
\hline Input & Acc & Diff from 3D CNN \\
\hline Binary Hand & 12.09 & +0.98 \\
3D Hand & 11.14 & -0.90 \\
Gray Hand & 12.86 & -1.36 \\
Color Hand & 16.84 & -1.69 \\
\hline
\end{tabular}

Table 2: Difference in subject recognition accuracy when swapping 3D convolution with 2D convolution.

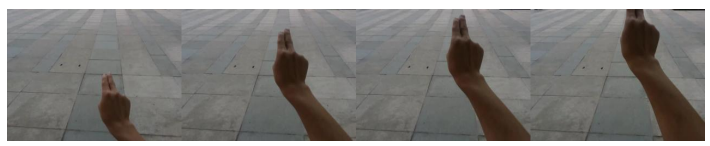

(a) Gesture 80. A gesture that is easy to recognize subject.

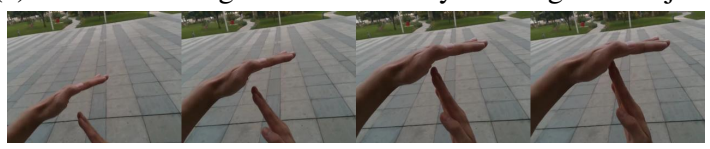

(b) Gesture 36. A gesture that is hard to recognize subject.

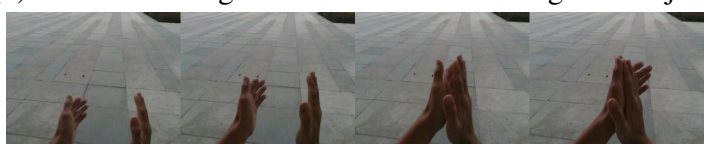

(c) Gesture 52. A gesture that is hard to recognize subject.

Figure 5: Sample gestures that are (a) easy and $(b, c)$ hard for recognizing subjects.

test on outdoor videos, the CNNs can overfit on background specific to the subjects.

To test this hypothesis, we perform experiments with class activation maps (CAM) [53] to visualize the image evidence used for making classification decisions. CAM is a linear combination of the feature maps produced by the last convolutional layer, with the linear coefficients proportional to the weight in the last classification layer. CAM highlights regions that the network is relying on. We show a sample clip each from training set and test set along with CAMs from RGB and Color Hand models in Figure 3 . As we expected, if the backgrounds are not removed, the classifier seems to cue on the background instead of hands. These are just two random samples, so in order to provide more robust results, we sample 1000 clips from the test set, compute the average CAM image, and compare it with the average image of binary hand masks. As shown in Figure 4, the handonly input produces an average CAM image whose peak is closer to the peak of the actual hand mask. This observation supports our hypothesis that raw RGB frames lead the model to overfit to the background, causing the test accuracy drop.

\subsubsection{Importance of Motion Information}

We use 3D convolutional networks for all experiments, so all models internally use the information of hand motion. However, it is interesting to measure how much motion information contributes to accuracy. To investigate this, we swap all 3D convolutions with 2D convolutions, extract the 2D CNN feature for each frame, and use the average feature vector as a representation of the clip. We compare the accuracy of this model with the 3D CNNs. Table 2 shows the results. For 3D Hand, Gray Hand, and Color Hand, the accuracy drops around 1 point, indicating the importance of motion information. However, to our surprise, the accuracy increases $0.98 \%$ for the input of Binary Hand. This indicates that when we only capture 2D hand shapes, considering the temporal feature is not helpful and possibly confusing. We speculate that because 2D hand shapes are essentially only edges of hands, they do not carry enough information to distinguish complex hand motion.

\subsubsection{Effect of Gesture Class}

We suspect that some gestures are more suitable to classifying subjects than others. Therefore, we investigate accuracy based on gesture types. This analysis is possible because the dataset was originally proposed for gesture recognition. We compute the subject recognition accuracy per gesture class and show it in Figure 10 in Supplementary Material. While the highest accuracy is $29.79 \%$ with gesture 80 , the lowest is $10.64 \%$ with gesture 36 and 52 . We show samples on gestures 80, 36, and 52 in Figure 5. The easy case (gesture 80) uses a single hand with the back of the hand clearly visible, while the difficult ones (gesture 36 and 52) use both hands with lateral views, making it harder to identify the subject.

\subsubsection{Effect of Clip Length}

Figure 6 shows a histogram of clip length in the test set. The shortest clip only has three frames while the longest has 122 frames. The mean length is 33.1 and the median is 32 . Does the length of clip affect the recognition accuracy? To investigate this, we divide the test set into short, medium, and long clips based on the 25 ( 26 frames) and 75 percentile (39 frames) of the length distribution. We use 3D Color Hand as input and show the accuracy based on this split in Table 3 The short, medium, and long clips have accuracies of $19.13 \%, 20.55 \%$, and $17.84 \%$, respectively. This result was not expected because we hypothesized that longer clips would be easier because they contain more information. We speculate that since the $\mathrm{CNN}$ is trained with a fixed-length input (16 consecutive frames), if the clip length is too long (longer than 39 - more than twice that of the fixed input lengths), the CNN cannot effectively capture key information compared to medium-length clips. 


\begin{tabular}{cccc}
\hline & Short & Medium & Long \\
\hline Accuracy & 19.13 & 20.55 & 17.84 \\
\hline
\end{tabular}

Table 3: Accuracy (\%) over the clip length for subject recognition. We divide the test clips into short, medium, and long with the boundaries of $25 \%$ quantile and $75 \%$ quantile.

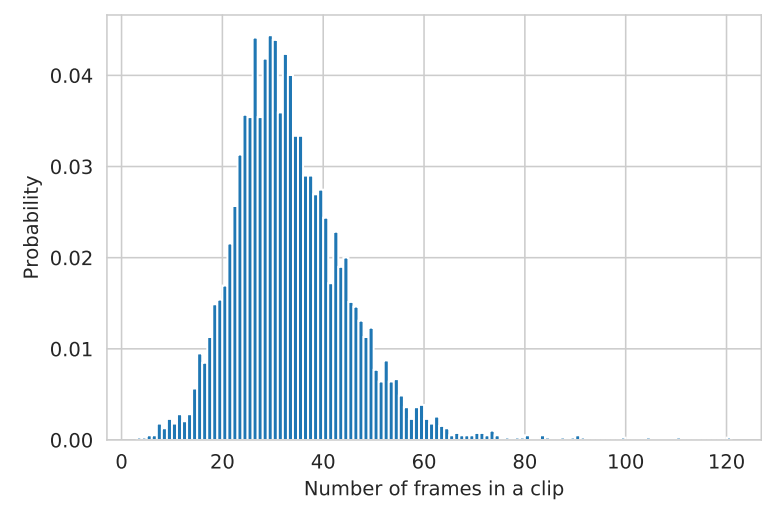

Figure 6: Histogram of number of frames per video in the EgoGesture test set.

\begin{tabular}{lcc}
\hline Input & Seen Gestures & Unseen Gestures (Diff) \\
\hline Binary Hand & 11.34 & $7.40(-3.94)$ \\
3D Hand & 11.49 & $10.24(-1.25)$ \\
Gray Hand & 14.82 & $12.01(-2.81)$ \\
Color Hand & 19.50 & $14.85(-4.65)$ \\
\hline
\end{tabular}

Table 4: Subject recognition accuracy (\%) drop for the seen and unseen gestures. We train the model with only half the available gestures and compute the test accuracy on seen and unseen gestures separately to see the generalization ability in terms of unseen hand pose.

\subsubsection{Subject Recognition for Unseen Gestures}

Our experiments so far divide the dataset based on its recorded place (indoor for training and outdoor for testing), and all gesture classes appear in both training and test sets. Another question is how well the model generalizes to unseen gestures. To answer this, we subsampled the training set by choosing half the gestures (exact split provided in the Supplementary Material), and compute test accuracy for seen and unseen gestures separately. As shown in Table 4 the accuracy drops by $3.94,1,25,2.81$, and 4.65 percentage points for Binary Hand, 3D Hand, Gray Hand, and Color Hand respectively. As expected, it becomes more difficult to recognize the subjects if the hands are in unseen poses.

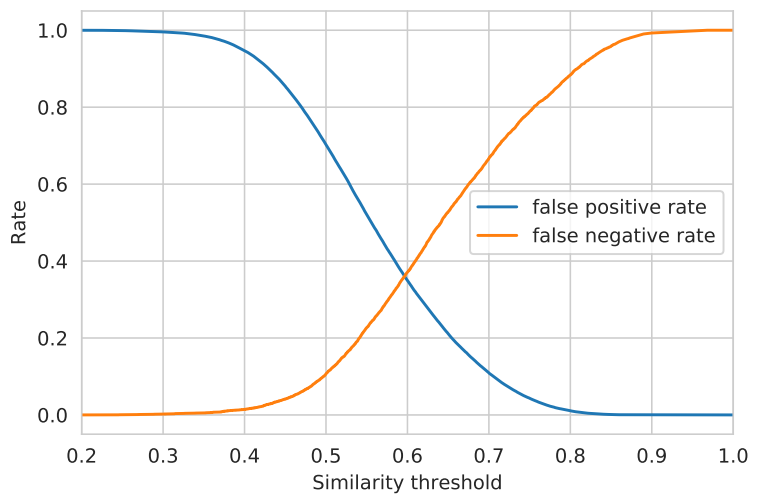

Figure 7: Trade-off between false positive rate and false negative rate for gesture-based user verification settings.

\subsubsection{Gesture Based Verification}

So far, the task is to recognize the subject that appeared in the training set, but a practical scenario could be user verification: given a pair of gesture clips, judge if they are from the same subject or not, even if the subject has not been seen before. To test this, we use 30 subjects for training, 10 for validation, and 10 for testing, and provide the exact split in Supplementary Material. With this split, we learn the representation by training on a classification task, but for evaluation, we perform gesture-based user verification by thresholding the cosine similarity of clip pairs. An evaluation pair consists of an indoor clip and an outdoor clip performing the same gesture. This amounts to 58,888 pairs of video clips with a heavy class imbalance, where only 5,950 pairs are positive. To incorporate this imbalance into the evaluation, we report Equal Error Rate (EER) where the threshold is set to have the same false-positive rate and false-negative rate. We evaluate with the input of 3D Color Hand and obtain an EER of $36.01 \%$. We plot the trade-off between false-positive rate and false-negative rate for different thresholds in Figure 7. We also plot the ROC curve in Figure 9 in Supplementary Material.

\subsubsection{Gesture Recognition Results}

In addition to recognizing the subject, our model potentially could benefit existing gesture recognition tasks as well. For these experiments, we use the data split defined in the original dataset where train/val/test have disjoint subjects because the model is expected to generalize to unseen people. We experiment with two ways to train the gesture recognition model with the auxiliary subject classifier as described in Sec. 3.2 and summarize the results in Table 5. The first way is to jointly train the gesture classifier and subject classifier while sharing the internal $\mathrm{CNN}$ representation as a multi-task learning task. Unfortunately, this method has an 


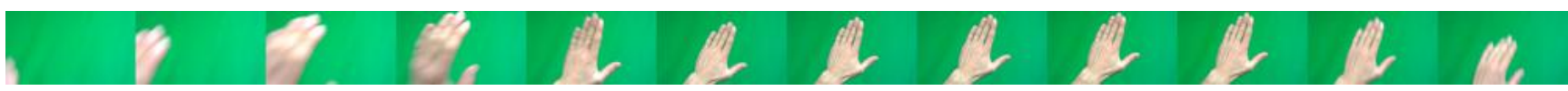

(1) Sample frames



(2) Extracted hand masks by removing the green chromakey background.

Figure 8: Green Gesture Dataset

\begin{tabular}{lcc}
\hline & RGB & Depth \\
\hline Gesture only & 88.42 & 89.35 \\
Joint with Subject-ID & 86.91 & 86.64 \\
Adversarial with Subject-ID & $\mathbf{8 9 . 5 1}$ & $\mathbf{8 9 . 6 6}$ \\
\hline
\end{tabular}

Table 5: Gesture recognition accuracy (\%) on EgoGesture dataset.

accuracy drop from the single-task training both for RGB (from $88.42 \%$ to $86.91 \%$ ) and for Depth (from $89.35 \%$ to $86.64 \%$ ). This suggests that the representations predictive of subjects are harmful to classify gestures, and the representations invariant to subjects are better. This is intuitive given that the gesture recognition model is expected to generalize to unseen subjects. Therefore we realize this representation with adversarial training and observe the accuracy improves both for RGB (from $88.42 \%$ to $89.51 \%$ ) and for Depth (from $89.35 \%$ to $89.66 \%$ ).

\subsection{Subject Recognition on GGesture Dataset}

We also perform subject classification experiments on the Green Gesture (GGesture) [9] dataset. This dataset has about 700 egocentric video clips of 10 gesture classes performed by 22 subjects. It has RGB clips without depth, and recorded only indoors. However, unlike the EgoGesture dataset, the background is always a green screen for chroma key composition, so we can easily extract the hand masks. Each subject has three rounds of recordings so we use them for train/val/test split, resulting in 229/216/221 clips, respectively. We show the results in Table 5 next to the EgoGesture results. Because GGesture does not have depth information, we only ablate the input with Binary Hand, Gray Hand, and Color Hand, which have accuracies of $51.13 \%, 61.09 \%$, and $64.71 \%$ respectively. The accuracy gain corresponds to the contribution of 2D shape of hands, skin texture, and skin colors, respectively.

\section{Discussion and Conclusion}

We have presented a CNN-based approach to recognize subjects from egocentric hand gestures. To our knowledge, this is the first study to show the potential to identify subjects based on ego-centric views of hands. We also perform ablative experiments to investigate the properties of input videos that contribute to the recognition performance. The experiments shows that hands shape in $2 \mathrm{D}$ and $3 \mathrm{D}$, skin texture, skin colors, and hand motions are all keys to identify a person's identity. Moreover, we also show that training gesture classifiers adversarially with subject identity recognition can improve the gesture recognition accuracy.

Our work has several limitations. First, while our hand masks remove the background and most other objects, it is possible that the model still cues on users' hand accessories (e.g. rings) to identify the user. We are aware that at least three subjects out of 50 in the EgoGesture dataset wear rings in some videos. Nevertheless, this is not an issue when we use depth modality or only hand shapes, and we note that the accuracy for those cases is greater than $10 \%$ over 50 subjects where random guess accuracy is only $2 \%$. Second, the performance is far from perfect, with a verification error rate of around $36 \%$, which means more than one out of every three verifications is wrong. However, this work is a first step in solving a new problem, and first approaches often have low performance and strong assumptions; object recognition 15 years ago was evaluated with six class classification - which was referred to as "six diverse object categories presenting a challenging mixture of visual characteristics" [13] — as opposed to the thousands today. Nonetheless, we experimentally showed that our task can benefit hand gesture recognition. We hope our work inspires more work in hand-based identity recognition, especially in the context of first-person vision.

Acknowledgments. This work was supported in part by the National Science Foundation (CAREER IIS-1253549) and by Indiana University through the Emerging Areas of Research Initiative Learning: Brains, Machines and Children.

\section{References}

[1] https://www.freepik.com/free-photo/surgeons-performingoperation-operation-room_1008439.htm.

[2] Mahmoud Afifi. 11k hands: gender recognition and biometric identification using a large dataset of hand images. Multimedia Tools and Applications, 2019. 
[3] Chetan Arora and Vivek Kwatra. Stabilizing first person 360 degree videos. In IEEE Winter Conference on Applications of Computer Vision (WACV), 2018.

[4] Sven Bambach, Stefan Lee, David J. Crandall, and Chen Yu. Lending a hand: Detecting hands and recognizing activities in complex egocentric interactions. In IEEE International Conference on Computer Vision, 2015.

[5] Salil P Banerjee and Damon L Woodard. Biometric authentication and identification using keystroke dynamics: A survey. Journal of Pattern Recognition Research, 7(1):116-139, 2012.

[6] Gedas Bertasius, Hyun Soo Park, X Yu Stella, and Jianbo Shi. Unsupervised learning of important objects from firstperson videos. In IEEE International Conference on Computer Vision, 2017.

[7] Guilherme Boreki and Alessandro Zimmer. Hand geometry: a new approach for feature extraction. In Fourth IEEE Workshop on Automatic Identification Advanced Technologies (AutoID'05), pages 149-154. IEEE, 2005.

[8] Congqi Cao, Yifan Zhang, Yi Wu, Hanqing Lu, and Jian Cheng. Egocentric gesture recognition using recurrent $3 \mathrm{~d}$ convolutional neural networks with spatiotemporal transformer modules. In IEEE International Conference on Computer Vision, 2017.

[9] Tejo Chalasani, Jan Ondrej, and Aljosa Smolic. Egocentric gesture recognition for head-mounted ar devices. In IEEE International Symposium on Mixed and Augmented Reality, 2018.

[10] Dima Damen, Hazel Doughty, Giovanni Maria Farinella, Sanja Fidler, Antonino Furnari, Evangelos Kazakos, Davide Moltisanti, Jonathan Munro, Toby Perrett, Will Price, and Michael Wray. Scaling egocentric vision: The epic-kitchens dataset. In European Conference on Computer Vision, 2018.

[11] John Daugman. Iris recognition border-crossing system in the uae. International Airport Review, 8(2), 2004.

[12] Jiankang Deng, Jia Guo, Niannan Xue, and Stefanos Zafeiriou. Arcface: Additive angular margin loss for deep face recognition. In IEEE Conference on Computer Vision and Pattern Recognition, 2019.

[13] Robert Fergus, Pietro Perona, Andrew Zisserman, et al. Object class recognition by unsupervised scale-invariant learning. In IEEE Conference on Computer Vision and Pattern Recognition, 2003.

[14] Thomas B Fitzpatrick. The validity and practicality of sunreactive skin types i through vi. Archives of dermatology, 124(6):869-871, 1988.

[15] Yaroslav Ganin and Victor Lempitsky. Unsupervised domain adaptation by backpropagation. In International Conference on Machine Learning, 2015.

[16] Guillermo Garcia-Hernando, Shanxin Yuan, Seungryul Baek, and Tae-Kyun Kim. First-person hand action benchmark with rgb-d videos and $3 \mathrm{~d}$ hand pose annotations. In IEEE Conference on Computer Vision and Pattern Recognition, 2018.

[17] Kensho Hara, Hirokatsu Kataoka, and Yutaka Satoh. Can spatiotemporal $3 \mathrm{~d}$ cnns retrace the history of $2 \mathrm{~d}$ cnns and imagenet? In IEEE Conference on Computer Vision and Pattern Recognition, 2018.
[18] Kaiming He, Xiangyu Zhang, Shaoqing Ren, and Jian Sun. Deep residual learning for image recognition. In IEEE Conference on Computer Vision and Pattern Recognition, 2016.

[19] Hsuan-I Ho, Wei-Chen Chiu, and Yu-Chiang Frank Wang. Summarizing first-person videos from third persons' points of view. In European Conference on Computer Vision, 2018.

[20] Yedid Hoshen and Shmuel Peleg. An egocentric look at video photographer identity. In IEEE Conference on Computer Vision and Pattern Recognition, 2016.

[21] Yifei Huang, Minjie Cai, Zhenqiang Li, and Yoichi Sato. Predicting gaze in egocentric video by learning taskdependent attention transition. In European Conference on Computer Vision, 2018.

[22] Anil K Jain, Patrick Flynn, and Arun A Ross. Handbook of biometrics. Springer Science \& Business Media, 2007.

[23] Hao Jiang and Kristen Grauman. Seeing invisible poses: Estimating $3 \mathrm{~d}$ body pose from egocentric video. In IEEE Conference on Computer Vision and Pattern Recognition, 2017.

[24] Diederik P Kingma and Jimmy Ba. Adam: A method for stochastic optimization. In International Conference on Learning Representations (ICLR), 2015.

[25] Ajay Kumar and Cyril Kwong. Towards contactless, lowcost and accurate $3 \mathrm{~d}$ fingerprint identification. In IEEE Conference on Computer Vision and Pattern Recognition, 2013.

[26] Kyungjun Lee, Abhinav Shrivastava, and Hernisa Kacorri. Hand-priming in object localization for assistive egocentric vision. In IEEE Winter Conference on Applications of Computer Vision, 2020.

[27] Yin Li, Miao Liu, and James M. Rehg. In the eye of beholder: Joint learning of gaze and actions in first person video. In European Conference on Computer Vision, 2018.

[28] Yang Liu, Ping Wei, and Song-Chun Zhu. Jointly recognizing object fluents and tasks in egocentric videos. In IEEE International Conference on Computer Vision, 2017.

[29] Davide Moltisanti, Michael Wray, Walterio W MayolCuevas, and Dima Damen. Trespassing the boundaries: Labeling temporal bounds for object interactions in egocentric video. In IEEE International Conference on Computer Vision, 2017.

[30] Franziska Mueller, Dushyant Mehta, Oleksandr Sotnychenko, Srinath Sridhar, Dan Casas, and Christian Theobalt. Real-time hand tracking under occlusion from an egocentric rgb-d sensor. In IEEE International Conference on Computer Vision, 2017.

[31] Katsuyuki Nakamura, Serena Yeung, Alexandre Alahi, and Li Fei-Fei. Jointly learning energy expenditures and activities using egocentric multimodal signals. In IEEE Conference on Computer Vision and Pattern Recognition, 2017.

[32] Nobuyuki Otsu. A threshold selection method from graylevel histograms. IEEE transactions on systems, man, and cybernetics, 9(1):62-66, 1979.

[33] Rohit Pandey, Pavel Pidlypenskyi, Shuoran Yang, and Christine Kaeser-Chen. Efficient 6-dof tracking of handheld objects from an egocentric viewpoint. In European Conference on Computer Vision, September 2018.

[34] Suvam Patra, Kartikeya Gupta, Faran Ahmad, Chetan Arora, and Subhashis Banerjee. Ego-slam: A robust monocular 
slam for egocentric videos. In IEEE Winter Conference on Applications of Computer Vision (WACV), 2019.

[35] Alessandro Penna, Sadegh Mohammadi, Nebojsa Jojic, and Vittorio Murino. Summarization and classification of wearable camera streams by learning the distributions over deep features of out-of-sample image sequences. In IEEE International Conference on Computer Vision, 2017.

[36] Yair Poleg, Chetan Arora, and Shmuel Peleg. Head motion signatures from egocentric videos. In Asian Conference on Computer Vision, pages 315-329. Springer, 2014.

[37] Rafael Possas, Sheila Pinto Caceres, and Fabio Ramos. Egocentric activity recognition on a budget. In IEEE Conference on Computer Vision and Pattern Recognition, 2018.

[38] Raul Sanchez-Reillo, Carmen Sanchez-Avila, and Ana Gonzalez-Marcos. Biometric identification through hand geometry measurements. IEEE Transactions on Pattern Analysis and Machine Intelligence, 22(10):1168-1171, 2000.

[39] Yang Shen, Bingbing Ni, Zefan Li, and Ning Zhuang. Egocentric activity prediction via event modulated attention. In European Conference on Computer Vision, 2018.

[40] Michel Silva, Washington Ramos, Joao Ferreira, Felipe Chamone, Mario Campos, and Erickson R Nascimento. A weighted sparse sampling and smoothing frame transition approach for semantic fast-forward first-person videos. In IEEE Conference on Computer Vision and Pattern Recognition, 2018.

[41] Linda B Smith, Swapnaa Jayaraman, Elizabeth Clerkin, and Chen Yu. The developing infant creates a curriculum for statistical learning. Trends in cognitive sciences, 22(4):325336, 2018.

[42] Randall Stross. Wearing a badge, and a video camera. The New York Times, 1, 2013.

[43] Hamed Rezazadegan Tavakoli, Esa Rahtu, Juho Kannala, and Ali Borji. Digging deeper into egocentric gaze prediction. In IEEE Winter Conference on Applications of Computer Vision, 2019.

[44] Daksh Thapar, Chetan Arora, and Aditya Nigam. Is sharing of egocentric video giving away your biometric signature? In European Conference on Computer Vision, 2020.

[45] Takeshi Uemori, Atsushi Ito, Yusuke Moriuchi, Alexander Gatto, and Jun Murayama. Skin-based identification from multispectral image data using cnns. In IEEE Conference on Computer Vision and Pattern Recognition, 2019.

[46] Aisha Urooj and Ali Borji. Analysis of hand segmentation in the wild. In IEEE Conference on Computer Vision and Pattern Recognition, 2018.

[47] Yanxiang Wang, Bowen Du, Yiran Shen, Kai Wu, Guangrong Zhao, Jianguo Sun, and Hongkai Wen. Ev-gait: Eventbased robust gait recognition using dynamic vision sensors. In IEEE Conference on Computer Vision and Pattern Recognition, 2019.

[48] Erdem Yoruk, Ender Konukoglu, Bülent Sankur, and Jérôme Darbon. Shape-based hand recognition. IEEE Transactions on Image Processing, 15(7):1803-1815, 2006.

[49] Ye Yuan and Kris Kitani. 3d ego-pose estimation via imitation learning. In European Conference on Computer Vision, 2018.
[50] Hasan FM Zaki, Faisal Shafait, and Ajmal S Mian. Modeling sub-event dynamics in first-person action recognition. In IEEE Conference on Computer Vision and Pattern Recognition, 2017.

[51] Mengmi Zhang, Keng Teck Ma, Joo-Hwee Lim, Qi Zhao, and Jiashi Feng. Deep future gaze: Gaze anticipation on egocentric videos using adversarial networks. In IEEE Conference on Computer Vision and Pattern Recognition, 2017.

[52] Yifan Zhang, Congqi Cao, Jian Cheng, and Hanqing Lu. Egogesture: a new dataset and benchmark for egocentric hand gesture recognition. IEEE Transactions on Multimedia, 20(5):1038-1050, 2018.

[53] Bolei Zhou, Aditya Khosla, Agata Lapedriza, Aude Oliva, and Antonio Torralba. Learning deep features for discriminative localization. In IEEE Conference on Computer Vision and Pattern Recognition, 2016. 


\section{Supplementary Material}

\subsection{Subject Recognition Accuracy per Gesture}

\begin{tabular}{|c|c|c|c|}
\hline & & Label Index & Accuracy \\
\hline Label Index & Accuracy & 56 & 19.15 \\
\hline 52 & 10.64 & 9 & 19.15 \\
\hline 36 & 10.64 & 69 & 19.15 \\
\hline 16 & 12.77 & 3 & 19.15 \\
\hline 68 & 12.77 & 6 & 19.15 \\
\hline 61 & 12.77 & 12 & 19.57 \\
\hline 14 & 12.77 & 78 & 19.57 \\
\hline 60 & 14.58 & 41 & 20.83 \\
\hline 45 & 14.89 & 81 & 20.83 \\
\hline 21 & 14.89 & 54 & 21.28 \\
\hline 17 & 14.89 & 55 & 21.28 \\
\hline 15 & 14.89 & 59 & 21.28 \\
\hline 31 & 14.89 & 58 & 21.28 \\
\hline 65 & 14.89 & 7 & 21.28 \\
\hline 71 & 14.89 & 74 & 21.28 \\
\hline 53 & 14.89 & 22 & 21.28 \\
\hline 77 & 15.22 & 27 & 21.28 \\
\hline 13 & 17.02 & 2 & 21.28 \\
\hline 43 & 17.02 & 38 & 21.28 \\
\hline 26 & 17.02 & 79 & 21.28 \\
\hline 25 & 17.02 & 67 & 23.40 \\
\hline 24 & 17.02 & 4 & 23.40 \\
\hline 23 & 17.02 & 76 & 23.40 \\
\hline 46 & 17.02 & 5 & 23.40 \\
\hline 49 & 17.02 & 72 & 23.40 \\
\hline 51 & 17.02 & 33 & 23.40 \\
\hline 63 & 17.02 & 64 & 23.40 \\
\hline 10 & 17.02 & 82 & 23.40 \\
\hline 75 & 17.02 & 47 & 23.40 \\
\hline 83 & 17.02 & 48 & 23.40 \\
\hline 73 & 17.39 & 44 & 23.40 \\
\hline 39 & 17.39 & 57 & 23.40 \\
\hline 42 & 17.39 & 18 & 23.40 \\
\hline 11 & 19.15 & 1 & 23.40 \\
\hline 19 & 19.15 & 70 & 23.91 \\
\hline 8 & 19.15 & 66 & 23.91 \\
\hline 30 & 19.15 & 20 & 25.53 \\
\hline 50 & 19.15 & 32 & 25.53 \\
\hline 62 & 19.15 & 35 & 25.53 \\
\hline 29 & 19.15 & 34 & 25.53 \\
\hline \multirow{2}{*}{40} & 19.15 & 28 & 27.66 \\
\hline & & $\begin{array}{l}37 \\
80\end{array}$ & $\begin{array}{l}27.66 \\
29.79\end{array}$ \\
\hline
\end{tabular}

Table 6: Subject recognition accuracy as a function of gesture class, sorted by accuracy. This is the corresponding data for Figure 10

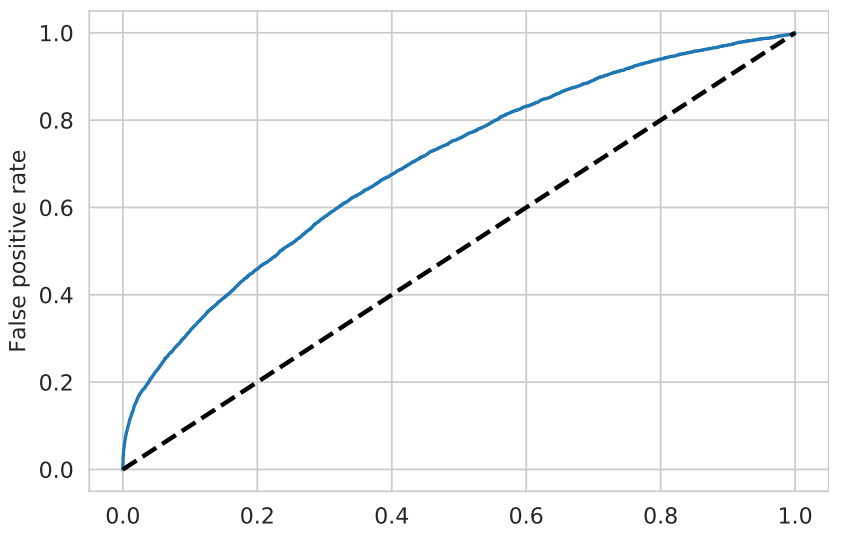

Figure 9: ROC curve for gesture-based user verification

\subsection{Dataset Split for Subject Recognition for Un- seen Gestures}

We select the following gestures for training: $2,4,6,8$, $10,12,14,16,18,20,22,24,26,28,30,32,34,36,38,40$, $42,44,46,48,50,52,54,56,58,60,62,64,66,68,70,72$, $74,76,78,80$, and 82 .

\subsection{Dataset Split for Gesture Based Verification}

We use 30 subjects for training, 10 for validation, and 10 for testing. The subjects for train is $3,4,5,6,8,10,15,16$, $17,20,21,22,23,25,26,27,30,32,36,38,39,40,42,43$, $44,45,46,48,49$, and 50 . The validation is $2,9,11,14,18$, $19,28,31,41$, and 47 . The testing is $1,7,12,13,24,29$, $33,34,35$, and 37 . 


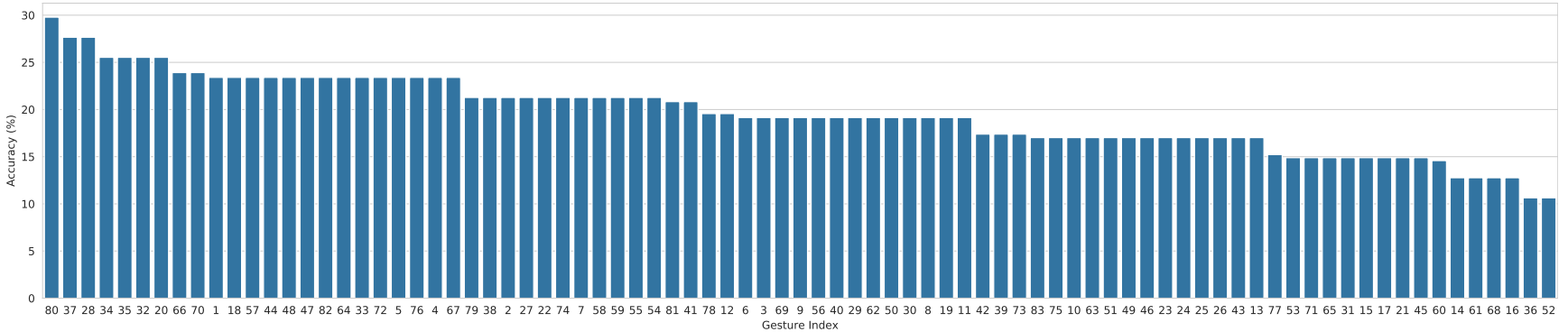

Figure 10: Subject recognition accuracy as a function of gesture class, sorted by accuracy. Some gestures allow subjects to be more easily recognized than others. Table in Supplementary Material shows the original data. 